\title{
Transaction Routing in Real-Time Shared Disks Clusters ${ }^{\star}$
}

\author{
Kyungoh Ohn, Sangho Lee, and Haengrae Cho \\ Department of Computer Engineering, Yeungnam University, \\ Gyungsan, Gyungbuk 712-749, Republic of Korea \\ hrcho@yu.ac.kr
}

\begin{abstract}
This paper proposes a real-time transaction routing algorithm, which allocates real-time transactions to a node in a shared disks cluster. Unlike traditional routing algorithms, which consider load balancing and transaction affinity only, our algorithm also considers transaction priorities inherent to real-time applications.
\end{abstract}

\section{Introduction}

Although there has been a great deal of independent research in real-time processing and shared disks (SD) cluster, their aggregation has very little attention 3. The cluster technology enables highly available real-time database services, which are the core of many telecommunication services. The cluster can also achieve high performance real-time transaction processing by exploiting internode parallelism.

This paper proposes a real-time transaction routing algorithm in the SD cluster. The transaction routing is a process of the front-end router to select an execution node for an incoming transaction. The traditional transaction routing algorithms of the SD cluster have two design goals: load balancing and transaction affinity 4, 5, 6. The load balancing means to avoid overloading individual node. The transaction affinity means to execute transactions with similar data access pattern on the same node (affinity node). To support real-time transactions, we have additional goal of transaction priority. This goal means to reduce the number of transactions missing their deadlines by considering the deadline as a priority. The contribution of this paper is to extend a well-performed traditional algorithm, named DACA (Dynamic Affinity Cluster Allocation) proposed by authors [4, to the real-time transaction processing.

\section{Algorithm}

We propose a new real-time transaction routing algorithm, named P-DACA ( Priority conscious $D A C A$ ). In this section, we first describe our underlying model

\footnotetext{
* This research was supported by University IT Research Center Project.
} 
and then summarize the basic idea of DACA algorithm [4. Lastly, we present P-DACA algorithm that extends DACA for real-time transaction processing.

\subsection{Assumption}

A transaction router selects an execution node of each incoming transaction. The node schedules the execution of its transactions with earliest deadline first (EDF) policy 11. We assume the mixed real-time transaction workload [2], which consists of real-time transactions and non real-time transactions. A real-time transaction is assigned with a deadline. Executing the real-time transaction after its deadline is meaningless.

We assume that the record-level locking is in effect. In real-time applications, the locking has to handle the priority inversion problem that lower priority transactions block the execution of higher priority transactions. To prevent the problem, we consider the locking model of [2. Specifically, a real-time transaction aborts non real-time transactions holding locks in conflict mode. On the other hand, a non real-time transaction always waits at lock conflict. The same procedure holds between real-time transactions with different priorities. High priority transactions are always guaranteed to acquire locks without waiting.

\section{$2.2 \quad$ DACA}

To alleviate the routing overhead, DACA considers balancing the load of each affinity cluster (AC) [5]. An AC collects several transaction classes with high affinity to a given set of tables. The transaction router maintains routing parameters. Specifically, when a transaction router allocates a transaction of an affinity cluster $\mathrm{AC}_{q}$ to a node $N_{p}$, it increments both $\# \mathrm{~T}\left(\mathrm{AC}_{q}\right)$ and $\# \mathrm{~T}\left(N_{p}\right)$, which means the number of active transactions at $\mathrm{AC}_{q}$ and at $N_{p}$ respectively. Both counters are decremented when a transaction commits or aborts.

There are two overload types: $A C$ overload and a node overload. The AC overload implies that transactions of an $\mathrm{AC}$ are rushed into the system. The node overload occurs when a node $N_{p}$ is allocated to several ACs and \#T $\left(N_{p}\right)$ is over average. DACA balances the load of each node according to the overload type. If $A C_{q}$ is overloaded, then DACA allocates more nodes to $A C_{q}$ by node expansion strategy. If there is no AC overload but $N_{p}$ is overloaded, then DACA distributes some ACs assigned to $N_{p}$ to other node by $A C$ distribution strategy.

DACA can make an optimal balance between the affinity-based routing and load balancing as follows. First, DACA tries to reduce the number of nodes allocated to the overloaded $\mathrm{AC}$ if the load deviation of each node is not significant. This allows DACA to reduce the frequency of inter-node cache invalidations. Next, DACA prohibits allocating both an overloaded AC and other ACs to a node. As a result, DACA can achieve high buffer hit ratio for the overloaded AC. Even though several non-overloaded ACs may be allocated to a single node, efficient handling of the overloaded $\mathrm{AC}$ is more important to improve the overall transaction throughput. 


\subsection{Priority Conscious DACA (P-DACA)}

Before describing the details of P-DACA, we first illustrate the problem of DACA when transactions have priorities. Example 1 shows the problem.

Example 1: Suppose there are two ACs $\left(A C_{1}, A C_{2}\right)$ and two nodes $\left(N_{1}, N_{2}\right)$. $N_{1}$ is an affinity node of $A C_{1}$ and executes a transaction $t_{1}$ of priority $100 . N_{2}$ is an affinity node of $A C_{2}$ and executes a transaction $t_{2}$ of priority 60 . At this time, suppose a new transaction $t_{3}$ of priority 70 arrives, and $t_{3}$ belongs to $A C_{1}$. Then DACA allocates $t_{3}$ to its affinity node $N_{1}$ if $N_{1}$ does not result in overload state. However, $t_{3}$ has lower priority than $t_{1}$ and cannot be executed until $t_{1}$ completes. So $t_{3}$ has a higher probability of missing its deadline. On the other hand, if $t_{3}$ is allocated to $N_{2}$, then it can be executed immediately.

The goal of P-DACA is (a) to reduce the number of transactions missing their deadlines, and (b) to take advantages of affinity clustering as DACA. To achieve this goal, P-DACA performs the following three basic steps sequentially to decide where a new real-time transaction $t_{r}$ will be routed to.

1. If there is an affinity node of $t_{r}$ where the priority of $t_{r}$ becomes the highest one, then allocate $t_{r}$ to that node.

2. If there is a non-affinity node of $t_{r}$ where the priority of $t_{r}$ becomes the highest one, then allocate $t_{r}$ to that node.

3 . If there is no node that can execute $t_{r}$ immediately, then allocate $t_{r}$ to one of its affinity nodes.

The basic steps can be optimized if the transaction router maintains a priority list for each node. The priority list is a sorted list in descending order of priorities of active real-time transactions at the node. Then a new real-time transaction $t_{r}$ has to be allocated to a node where $t_{r}$ can be executed faster than other nodes. P-DACA checks this condition by comparing the relative position of $t_{r}$ in the priority list of each node. Suppose $P\left(t_{r}\right)$ means the priority of $t_{r}$, and $t_{r}$ is included in the affinity cluster $A C_{r} . \mathcal{S}\left(A C_{r}\right)$ is a set of affinity nodes of $A C_{r}$. The followings summarize the transaction routing algorithm of P-DACA.

1. $\# \mathrm{~T}\left(A C_{r}\right)=\# \mathrm{~T}\left(A C_{r}\right)+1$;

2. If ( $t_{r}$ is a real-time transaction) then

(a) If $\left(\exists N_{p} \in \mathcal{S}\left(A C_{r}\right), \operatorname{rank}\left(P\left(t_{r}\right), N_{p}\right)<w_{1} \operatorname{AND} \operatorname{rank}\left(P\left(t_{r}\right), N_{p}\right)<\right.$ $\operatorname{rank}\left(P\left(t_{r}\right), N_{i}\right)$, for all $\left.N_{i} \in \mathcal{S}\left(A C_{r}\right), i \neq p\right)$ then goto step 4;

(b) Else if $\left(\exists N_{p} \notin \mathcal{S}\left(A C_{r}\right), \operatorname{rank}\left(P\left(t_{r}\right), N_{p}\right)<w_{2}\right.$ AND $\operatorname{rank}\left(P\left(t_{r}\right), N_{p}\right)<$ $\operatorname{rank}\left(P\left(t_{r}\right), N_{i}\right)$, for all $\left.N_{i} \notin \mathcal{S}\left(A C_{r}\right), i \neq p\right)$ then goto step 5;

(c) Else goto step 3.

3. Select $N_{p}$, where $\# \mathrm{~T}\left(N_{p}\right)$ is minimum for all $N_{i} \in \mathcal{S}\left(A C_{r}\right)$;

4. If $\left(A C_{r}\right.$ is overloaded) then call node_expansion $\left(A C_{r}\right)$;

5. Else if $\left(N_{p}\right.$ is overloaded) then call ac_distribution $\left(N_{p}\right)$;

6. $\# \mathrm{~T}\left(N_{p}\right)=\# \mathrm{~T}\left(N_{p}\right)+1$; Insert $P\left(t_{r}\right)$ into the priority list of $N_{p}$;

7. Return $N_{p}$. 
At step 2, the function of $\operatorname{rank}\left(P\left(t_{r}\right), N_{p}\right)$ returns the number of transactions whose priorities are higher than $P\left(t_{r}\right)$ at $N_{p}$. If the function returns $0, t_{r}$ will be a transaction with the highest priority at $N_{p}$. The values $w_{1}$ and $w_{2}$ are windowing constraints that limit the acceptable rank of $t_{r} . w_{1}$ is usually bigger than $w_{2}$ since an affinity node of $t_{r}$ could complete $t_{r}$ faster. Note that if $w_{1}$ and $w_{2}$ are set to 1 , the algorithm works similarly to the above basic steps. A non real-time transaction is allocated to one of its affinity nodes with the lightest load (step 3). If allocating $t_{r}$ would cause an AC overload or a node overload, then the resolution strategy of DACA has to be performed (step 4 and 5). Example 2 shows how P-DACA can resolve the problem of Example 1.

Example 2: Suppose that the information of ACs, nodes, and transactions are same to Example 1. Suppose also that both $w_{1}$ and $w_{2}$ are set to 1 . Then the transaction router allocates $t_{3}$ to $N_{2}$ that can execute $t_{3}$ immediately. This is because (a) the affinity node of $t_{3}$ is $N_{1}$ but $\operatorname{rank}\left(P\left(t_{3}\right), N_{1}\right)=1=w_{1}$, and (b) even though $N_{2}$ is not an affinity node of $t_{3} \operatorname{but} \operatorname{rank}\left(P\left(t_{3}\right), N_{2}\right)=0<w_{2}$. Note that if $w_{1}$ is set to $2, t_{3}$ is allocated to $N_{1}$.

\section{Concluding Remarks}

We proposed a new transaction routing algorithm for the real-time SD cluster, named P-DACA. The notable features of P-DACA are two-fold. First, P-DACA allocates a real-time transaction to a node that guarantees higher probability of completing the transaction within its deadline. Even though the transaction could miss its deadline due to succeeding transactions with higher priority, the selection strategy is the best choice at the current state. Next, P-DACA tries to allocate a transaction to its affinity node if possible. So P-DACA can achieve high buffer hit ratio. This reduces the transaction execution time, and the probability of missing deadline can be reduced as a result.

\section{References}

1. Lam, K-Y., Kuo, T-W. (ed.): Real-Time Database Systems: Architecture and Techniques. Kluwer Academic Publishers (2000)

2. Lam, K-Y., Kuo, T-W., Lee, T.: Strategies for resolving inter-class data conflicts in mixed real-time database systems. Journal of Syst. and Soft. 61 (2002) 1-14

3. Lee, S., Ohn, K., Cho, H.: Feasibility and Performance Study of a Shared Disks Cluster for Real-Time Processing. Lecture Notes in Computer Science 3397 (2005) 518-527

4. Ohn, K., Cho, H.: Cache Conscious Dynamic Transaction Routing in a Shared Disks Cluster. Lecture Notes in Computer Science 3045 (2004) 548-557

5. Yu, P., Dan, A.: Performance Analysis Clustering on Transaction Processing Coupling Architecture. IEEE Trans. Knowledge and Data Eng. 6 (1994) 764-786

6. Yu, P., Dan, A.: Performance Evaluation of Transaction Processing Coupling Architectures for Handling System Dynamics. IEEE Trans. Parallel and Distributed Syst. 5 (1994) 139-153 